\title{
SOLUTION OF A CLASS OF SINGULAR INTEGRAL EQUATIONS
}

\author{
ERIC REISSNER
}

The following class of integral equations may be of some importance in the applications:

$$
g(x)=\frac{1}{\pi} \oint_{-1}^{1} f(\xi)\left\{\frac{1}{\xi-x}+\sum_{n=0}^{N} c_{n}(\xi-x)^{2 n+1}\right\} d \xi .
$$

The symbol $\Phi$ indicates that the principal value of the integral is to be taken and the coefficients $c_{n}$ are given constants. The special case of all $c_{n}=0$ has been dealt with extensively, for instance by Glauert [1], Fuchs [2], Hamel [3], Schroeder [4] and Söhngen [5]. ${ }^{1}$ The values of the coefficients $c_{n}$ might be determined by the condition that a given kernel $K(\xi-x)$, for instance $K=1 / \sinh (\xi-x)$, is approximated as nearly as possible by the kernel of equation (1).

The purpose of the present note is to derive the solution of (1) for a finite number of nonvanishing $c_{n}$. The method of solution is an extension of the method applicable when all $c_{n}=0$.

Equation (1) is first transformed by the substitutions

$$
\begin{aligned}
x & =\cos \phi, & \xi & =\cos \theta, \\
g(x) & =G(\phi), & f(\xi) & =F(\theta)
\end{aligned}
$$

into

(4) $G(\phi)=\frac{1}{\pi} \oint_{0}^{\pi} F(\theta)\left\{\frac{1}{\cos \theta-\cos \phi}+\sum_{n=0}^{N} c_{n}(\cos \theta-\cos \phi)^{2 n+1}\right\} \sin \theta d \theta$.

The function $G(\phi)$ is thought to be developed in the interval $(0, \pi)$ in the following form:

$$
\sin \phi G(\phi)=\sum_{m=1}^{\infty} B_{m} \sin m \phi .
$$

It is then to be shown that the following representation of $F(\theta)$

$$
\sin \theta F(\theta)=\sum_{m=0}^{\infty} A_{m} \cos m \theta
$$

permits the explicit determination of the unknown coefficients $A_{m}$ in

Received by the editors May 5, 1945.

${ }_{1}^{1}$ Numbers in brackets refer to the references cited at the end of the paper. 
terms of the known coefficients $B_{m}$ and $c_{n}$.

Substituting equations (5) and (6) in equation (4) there occur these integrals

$$
\begin{gathered}
\frac{1}{\pi} \oint_{0}^{\pi} \frac{\cos m \theta d \theta}{\cos \theta-\cos \phi}=\frac{\sin m \phi}{\sin \phi} \\
\frac{1}{\pi} \int_{0}^{\pi} \cos m \theta(\cos \theta-\cos \phi)^{2 n+1} d \theta=\sum_{k=1}^{2 n+2} D_{k}(m, n) \frac{\sin k \phi}{\sin \phi}
\end{gathered}
$$

Equation (7) may be found in reference [1]. The validity of equation (8) with suitable coefficients $D_{k}$ follows from the fact that its left side may be written as a polynomial of degree $2 n+1$ in $\cos \phi$ and therefore also as a series of the form $\sum_{j=0}^{2 n+1} a_{j} \cos j \theta$. It is important to note that the coefficients $D_{k}$ satisfy the following conditions,

$$
D_{k}(m, n)=0, \quad m=2 n+2,2 n+3, \cdots ; k=2 n+3,2 n+4, \cdots .
$$

On the basis of equations (5) to (8) equation (4) takes on the form

$$
\sum_{m=1}^{\infty} B_{m} \sin m \phi=\sum_{m=1}^{\infty} A_{m}\left\{\sin m \phi+\sum_{n=0}^{N} c_{n}\left[\sum_{k=1}^{2 n+2} D_{k}(m, n) \sin k \phi\right]\right\}
$$

This is equivalent to the following set of simultaneous equations for the quantities $A_{m}$,

$$
B_{j}=A_{j}+\sum_{m=1}^{\infty} A_{m}\left\{\sum_{n=0}^{N} c_{n} D_{j}(m, n)\right\}, \quad j=1,2, \cdots .
$$

But in view of equations (9) the system (11) may be written as

$$
\begin{aligned}
& B_{j}=A_{j}, \quad j=2 N+3,2 N+4, \cdots \\
& B_{j}=A_{j}+\sum_{m=1}^{2 N+1} A_{m}\left\{\sum_{n=0}^{N} c_{n} D_{j}(m, n)\right\}, \quad j=1,2, \cdots, 2 N+2 .
\end{aligned}
$$

Thus, it remains to solve a simultaneous system of $2 N+2$ equations for the $2 N+3$ unknowns $A_{0}, A_{1}, \cdots, A_{2 N+2}$. In analogy to the procedure when all $c_{n}=0$ we may express $A_{1}, \cdots, A_{2 N+2}$ in terms of $A_{0}$ and leave $A_{0}$ arbitrary or determine it by an extraneous condition such as for instance $F(0)=\sum_{0}^{\infty} A_{m}=0$.

It is to be underlined that the above reduction of the problem to a system of simultaneous equations for a finite number of unknowns depends on the fact that the regular part of the kernel in equation (1) consists of a polynomial and not of an infinite series. It is further to be noted that there may be critical values of the coefficients $c_{n}$ for 
which equations (12) have a solution only if certain relations between the quantities $B_{j}$ hold.

The following example may illustrate the foregoing results. Taking $N=1$ one finds, in this special case most easily directly from equations (4) to (7), that equations (12) become

$$
\begin{aligned}
& B_{1}=\left(1+\frac{c_{0}}{2}+\frac{3 c_{1}}{4}\right) A_{1}+\frac{c_{1}}{8} A_{3}, \\
& B_{2}=\left(1-\frac{3 c_{1}}{8}\right) A_{2}-\left(\frac{c_{0}}{2}+c_{1}\right) A_{0}, \\
& B_{3}=A_{3}+\frac{3 c_{1}}{8} A_{1}, B_{4}=A_{4}-\frac{c_{1}}{8} A_{0}, B_{j}=A_{j}, j=5,6, \cdots .
\end{aligned}
$$

Solving for $A_{j}$, we obtain

$$
\begin{aligned}
& A_{1}=\frac{B_{1}-c_{1} B_{3} / 8}{1+c_{0} / 2+3 c_{1} / 4-3 c_{1}^{2} / 64}, \quad A_{2}=\frac{B_{2}+\left(c_{0} / 2+c_{1}\right) A_{0}}{1-3 c_{1} / 8}, \\
& A_{3}=\frac{\left(1+c_{0} / 2+3 c_{1} / 4\right) B_{3}-3 c_{1} B_{1} / 8}{1+c_{0} / 2+3 c_{1} / 4-3 c_{1}^{2} / 64} \\
& A_{4}=B_{4}+\frac{c_{1}}{8} A_{0}, \quad A_{5}=B_{5}
\end{aligned}
$$

and so on.

Evidently, exceptional conditions exist when one or both denominators in equation (14) have the value zero. The meaning of this occurrence is that under those circumstances equation (4) has solutions of one or both of the forms $F=\cos 2 \theta / \sin \theta, F=(\cos \theta-\alpha \cos 3 \theta) / \sin \theta$ when $G=0$.

\section{REFERENCES}

1. H. Glauert, Aerofoil and airscrew theory, New York, 1943, pp. 87-93.

2. R. Fuchs, Theorie der Luftkräfte, Berlin, 1935, pp. 54-60.

3. G. Hamel, Integralgleichungen, Berlin, 1937, pp. 145-148.

4. K. Schröder, Über eine Integralgleichung erster Art der Tragflïgeltheorie, Preussischen Akademie der Wissenschaften, 1938, pp. 345-362.

5. H. Söhngen, Die Lösungen der Integralgleichung $g(x)=1 / 2 \pi \oint_{-1}^{1} f(\xi) /(x-\xi) d \xi$ und deren Anwendung in der Tragfliugeltheorie, Math. Zeit. vol. 45 (1939) pp. 245-264.

Massachusetrs Institute of Technology 